\title{
UCRL-TR-219523
}

LAWRENCE LIVERMORE N A T IO N A L LABORATORY
A plasticity integration algorithm motivated by analytical integration of a generalized quadratic function

R. Becker

March 6, 2006 
This document was prepared as an account of work sponsored by an agency of the United States Government. Neither the United States Government nor the University of California nor any of their employees, makes any warranty, express or implied, or assumes any legal liability or responsibility for the accuracy, completeness, or usefulness of any information, apparatus, product, or process disclosed, or represents that its use would not infringe privately owned rights. Reference herein to any specific commercial product, process, or service by trade name, trademark, manufacturer, or otherwise, does not necessarily constitute or imply its endorsement, recommendation, or favoring by the United States Government or the University of California. The views and opinions of authors expressed herein do not necessarily state or reflect those of the United States Government or the University of California, and shall not be used for advertising or product endorsement purposes.

This work was performed under the auspices of the U.S. Department of Energy by University of California, Lawrence Livermore National Laboratory under Contract W-7405-Eng-48. 


\section{A plasticity integration algorithm motivated by analytical integration of a generalized quadratic function}

The goal is to examine the dependence of the plastic flow direction as a function of strain increment for a generalized quadratic flow potential; and from that, extract a scheme for constructing a plastic flow direction for a more general class of yield and flow surfaces.

The associative generalized quadratic yield function is written as

$$
\phi=\psi=\sqrt{\frac{3}{2} \boldsymbol{\sigma}^{\prime}: \mathcal{K}: \boldsymbol{\sigma}^{\prime}}-\bar{\sigma}
$$

where $\bar{\sigma}$ is the material flow strength, $\mathcal{K}$ the fourth order anisotropy tensor, and $\phi$ and $\psi$ are, respectively, the yield and plastic flow potentials. The plastic flow normal to the flow surface is given by

$$
\mathbf{d}^{p}=\dot{\lambda} \frac{\partial \psi}{\partial \boldsymbol{\sigma}}=\dot{\lambda} \frac{3}{2} \frac{\mathcal{K}: \boldsymbol{\sigma}^{\prime}}{\bar{\sigma}}
$$

and from plastic work equivalence

$$
\boldsymbol{\sigma}: \mathbf{d}^{p}=\dot{\lambda} \frac{3}{2} \frac{\boldsymbol{\sigma}^{\prime}: \mathcal{K}: \boldsymbol{\sigma}^{\prime}}{\bar{\sigma}}=\dot{\lambda} \bar{\sigma}=\dot{\bar{\varepsilon}} \bar{\sigma}
$$

leading to $\dot{\lambda}=\dot{\bar{\varepsilon}}$.

The deviatoric part of the Jaumann stress rate is then given by

$$
\begin{aligned}
\hat{\boldsymbol{\sigma}}^{\prime} & =\mathcal{L}: \mathbf{d}^{e}=\mathcal{L}:\left(\mathbf{d}-\mathbf{d}^{p}\right) \\
& =\mathcal{L}: \mathbf{d}-\frac{3}{2} \frac{\dot{\bar{\varepsilon}}}{\bar{\sigma}} \mathcal{L}: \mathcal{K}: \boldsymbol{\sigma}^{\prime}
\end{aligned}
$$

All of the above follows standard procedures for determining rate equations for plasticity. This works nicely for small strain increments. However, when the strain increments are more than a few percent of the elastic strain, the plastic flow direction changes over the time step, and this must be factored into the integration scheme.

For isotropic J2 plasticity, the radial return algorithm sets the plastic flow direction aligned with the stress at the end of the time step. This provides an unconditionally stable solution, but the scheme is only first order accurate in the plastic flow direction and any effects of path change over the time increment are lost. A suitable generalization of the radial return algorithm for anisotropic materials has not been found. Even for the simple quadratic model shown above, a consistent implicit approach would involve inversions of a fourth order tensor inside an iteration loop.

The approach taken here is to integrate the stress evolution equation analytically over the time increment and characterize the direction of plastic flow as a function of the applied strain increment. The applied strain rate, the equivalent plastic strain rate and the flow strength are assumed to be constant over the time increment. This is consistent with standard integration schemes over a time step. The integration is accomplished by a forward integration scheme over a number of sub-increments, $N$, that approaches infinity. 
This laborious approach is to circumvent justifying how fourth order tensors are integrated into exponentials. The stress at sub-increment $M$ of the $N$ is

$$
\left[\boldsymbol{\sigma}_{t+\frac{M}{N} \Delta t}^{\prime}\right]=\left[\boldsymbol{\sigma}_{t+\frac{M-1}{N} \Delta t}^{\prime}\right]+\mathcal{L}: \mathbf{d} \frac{1}{N} \Delta t-\frac{3}{2} \frac{\dot{\bar{\varepsilon}}}{\bar{\sigma}} \mathcal{L}: \mathcal{K}:\left[\boldsymbol{\sigma}_{t+\frac{M-1}{N} \Delta t}^{\prime}\right] \frac{1}{N} \Delta t
$$

Applying this formula successively

$$
\begin{aligned}
& \boldsymbol{\sigma}_{t+\frac{\Delta t}{N}}^{\prime}=\left\{\mathcal{I}-\frac{3}{2} \frac{\dot{\bar{\varepsilon}}}{\overline{\bar{\sigma}}} \frac{\Delta t}{N} \mathcal{L}: \mathcal{K}\right\}: \boldsymbol{\sigma}_{t}^{\prime}+\mathcal{L}: \mathbf{d} \frac{\Delta t}{N} \\
& \boldsymbol{\sigma}_{t+\frac{2 \Delta t}{N}}^{\prime}=\left\{\mathcal{I}-\frac{3}{2} \frac{\dot{\bar{\varepsilon}}}{\bar{\sigma}} \frac{\Delta t}{N} \mathcal{L}: \mathcal{K}\right\}^{2}: \boldsymbol{\sigma}_{t}^{\prime}+\left\{\mathcal{I}-\frac{3}{2} \frac{\dot{\bar{\varepsilon}}}{\overline{\bar{\sigma}}} \frac{\Delta t}{N} \mathcal{L}: \mathcal{K}\right\}: \mathcal{L}: \mathbf{d} \frac{\Delta t}{N}+\mathcal{L}: \mathbf{d} \frac{\Delta t}{N} \\
& \boldsymbol{\sigma}_{t+\frac{3 \Delta t}{N}}^{\prime}=\left\{\mathcal{I}-\frac{3}{2} \frac{\dot{\bar{\varepsilon}}}{\overline{\bar{\sigma}}} \frac{\Delta t}{N} \mathcal{L}: \mathcal{K}\right\}^{3}: \boldsymbol{\sigma}_{t}^{\prime}+\left\{\mathcal{I}-\frac{3}{2} \frac{\dot{\bar{\varepsilon}}}{\overline{\bar{\sigma}}} \frac{\Delta t}{N} \mathcal{L}: \mathcal{K}\right\}^{2}: \mathcal{L}: \mathbf{d} \frac{\Delta t}{N} \\
& +\left\{\mathcal{I}-\frac{3}{2} \frac{\dot{\bar{\varepsilon}}}{\bar{\sigma}} \frac{\Delta t}{N} \mathcal{L}: \mathcal{K}\right\}: \mathcal{L}: \mathbf{d} \frac{\Delta t}{N}+\mathcal{L}: \mathbf{d} \frac{\Delta t}{N} \\
& \text {... } \\
& \boldsymbol{\sigma}_{t+\Delta t}^{\prime}=\left\{\mathcal{I}-\frac{3}{2} \frac{\dot{\bar{\varepsilon}}}{\bar{\sigma}} \frac{\Delta t}{N} \mathcal{L}: \mathcal{K}\right\}^{N}: \boldsymbol{\sigma}_{t}^{\prime}+\left[\sum_{S=0}^{N-1}\left\{\mathcal{I}-\frac{3}{2} \frac{\dot{\bar{\varepsilon}}}{\bar{\sigma}} \frac{\Delta t}{N} \mathcal{L}: \mathcal{K}\right\}^{S}\right]: \mathcal{L}: \mathbf{d} \frac{\Delta t}{N}
\end{aligned}
$$

Considerable simplification is possible. Writing the quantity in braces as

$$
\left\{\mathcal{I}-\frac{3}{2} \frac{\dot{\bar{\varepsilon}}}{\bar{\sigma}} \frac{\Delta t}{N} \mathcal{L}: \mathcal{K}\right\}=\mathcal{A}=\mathcal{I}-\frac{1}{N} \mathcal{B}
$$

to shorten the notation, expanding the binomial

$$
\mathcal{A}^{N}=\mathcal{I}-\frac{N}{N} \mathcal{B}+\frac{N(N-1)}{N^{2} 2 !} \mathcal{B}^{2}-\frac{N(N-1)(N-2)}{N^{3} 3 !} \mathcal{B}^{3}+\ldots
$$

and letting $N$ approach infinity gives

$$
\lim _{N \rightarrow \infty} \mathcal{A}^{N}=\lim _{N \rightarrow \infty}\left\{\mathcal{I}-\frac{1}{N} \mathcal{B}\right\}^{N}=\exp (-\mathcal{B})=\exp \left(-\frac{3}{2} \frac{\dot{\bar{\varepsilon}} \Delta t}{\bar{\sigma}} \mathcal{L}: \mathcal{K}\right)
$$

The simplified form for the summation is much the same as for scalars

$$
\frac{1}{N} \sum_{S=0}^{N-1} \mathcal{A}^{S}=\frac{1}{N}\left(\mathcal{I}-\mathcal{A}^{N}\right):(\mathcal{I}-\mathcal{A})^{-1}=\left(\mathcal{I}-\mathcal{A}^{N}\right): \mathcal{B}^{-1}
$$

This can be verified by multiplying the equation above by $(\mathcal{I}-\mathcal{A})$. It is also important to note that the inverse can be applied to the left or right side of $\left(\mathcal{I}-\mathcal{A}^{N}\right)$. Taking the limit as $N$ approaches infinity and using the result from Eq (9) 


$$
\lim _{N \rightarrow \infty} \frac{1}{N} \sum_{S=0}^{N-1} \mathcal{A}^{S}=\frac{2}{3} \frac{\bar{\sigma}}{\dot{\bar{\varepsilon}} \Delta t}\left[\mathcal{I}-\exp \left(-\frac{3}{2} \frac{\dot{\bar{\varepsilon}} \Delta t}{\bar{\sigma}} \mathcal{L}: \mathcal{K}\right)\right]:(\mathcal{L}: \mathcal{K})^{-1}
$$

The resulting equation for the stress at the end of the time increment is

$$
\boldsymbol{\sigma}_{t+\Delta t}^{\prime}=\exp \left(-\frac{3}{2} \frac{\dot{\bar{\varepsilon}} \Delta t}{\bar{\sigma}} \mathcal{L}: \mathcal{K}\right): \boldsymbol{\sigma}_{t}^{\prime}+\frac{2}{3} \frac{\bar{\sigma}}{\dot{\bar{\varepsilon}} \Delta t}\left[\mathcal{I}-\exp \left(-\frac{3}{2} \frac{\dot{\bar{\varepsilon}} \Delta t}{\bar{\sigma}} \mathcal{L}: \mathcal{K}\right)\right]: \mathcal{K}^{-1}: \mathbf{d} \Delta t
$$

The small and large strain increment limits are examined as a sanity check on the relation. For the behavior at small strain increments the quadratic and higher terms from the exponentials are neglected leaving

$$
\boldsymbol{\sigma}_{t+\Delta t}^{\prime}=\boldsymbol{\sigma}_{t}^{\prime}-\frac{3}{2} \frac{\dot{\bar{\varepsilon}} \Delta t}{\bar{\sigma}} \mathcal{L}: \mathcal{K}: \boldsymbol{\sigma}_{t}^{\prime}+\mathcal{L}: \mathbf{d} \Delta t
$$

which is the simple forward integration result from the rate equation, Eq (4). At strains much larger than the elastic limit strain the exponentials approach zero leaving

$$
\boldsymbol{\sigma}_{t+\Delta t}^{\prime}=\frac{2}{3} \frac{\bar{\sigma}}{\dot{\bar{\varepsilon}}} \mathcal{K}^{-1}: \mathbf{d}
$$

Moving $\mathcal{K}$ and the numerical constants to the left side of the equation and multiplying through by $\boldsymbol{\sigma}_{t+\Delta t}^{\prime}$ produces the square of the equivalent stress on the left side. Plastic work equivalence then suggests that the plastic part of the rate of deformation tensor approaches $\mathbf{d}$ at large strain increments, which must be the case since elastic strains are limited by the material strength.

More can be done with Eq (12) to produce closed form expressions for the energy dissipated and consistent tangent moduli for the generalized quadratic function. However, the goal here is to look beyond the generalized quadratic relation and glean something useful for integrating arbitrary flow relations. To this end, the direction of the normal to the flow potential (plastic flow direction) at the end of the time step can be constructed from $\mathrm{Eq}$ (12) after reordering the products in Eq (10)

$$
\begin{aligned}
\alpha \mathbf{N}_{t+\Delta t}=\frac{3}{2} \frac{\mathcal{K}: \boldsymbol{\sigma}_{t+\Delta t}^{\prime}}{\bar{\sigma}}= & \frac{3}{2} \mathcal{K}: \exp \left(-\frac{3}{2} \frac{\dot{\bar{\varepsilon}} \Delta t}{\bar{\sigma}} \mathcal{L}: \mathcal{K}\right): \frac{\boldsymbol{\sigma}_{t}^{\prime}}{\bar{\sigma}} \\
& +\mathcal{K}:(\mathcal{L}: \mathcal{K})^{-1}:\left[\mathcal{I}-\exp \left(-\frac{3}{2} \frac{\dot{\bar{\varepsilon}} \Delta t}{\bar{\sigma}} \mathcal{L}: \mathcal{K}\right)\right]: \mathcal{L}: \frac{\mathbf{d} \Delta t}{\overline{\dot{\varepsilon}} \Delta t}
\end{aligned}
$$

If the exponentials are expanded in a Taylor series, it is readily seen that $\mathcal{L}_{\mathrm{s}}$ and $\mathcal{K}_{\mathrm{s}}$ can be absorbed and kicked out of the individual terms of the expansion to arrive at

$$
\alpha \mathbf{N}_{t+\Delta t}=\frac{3}{2} \exp \left(-\frac{3}{2} \frac{\dot{\bar{\varepsilon}} \Delta t}{\bar{\sigma}} \mathcal{K}: \mathcal{L}\right): \mathcal{K}: \frac{\boldsymbol{\sigma}_{t}^{\prime}}{\bar{\sigma}}+\left[\mathcal{I}-\exp \left(-\frac{3}{2} \frac{\dot{\bar{\varepsilon}} \Delta t}{\bar{\sigma}} \mathcal{K}: \mathcal{L}\right)\right]: \frac{\mathbf{d} \Delta t}{\dot{\bar{\varepsilon}} \Delta t}
$$


Equation (16) shows the evolution of the flow potential normal as a function of the plastic strain increment. If the increment is very small, the normal is the same as at the beginning of the time increment. At large plastic strain increments the flow potential normal, and hence the plastic flow direction, is in direction of the applied strain increment. For intermediate plastic strain increments the normal is some weighted average of the two limits. This is not necessarily a simple weighting of the two limiting directions as the fourth order tensors in the argument of the exponential will cause some deviation.

\section{Proposed integration scheme}

Using Eq (16) as motivation, it is proposed that the flow potential normal at the end of a time increment be approximated as a simple weighting of the two limiting directions.

$$
\mathbf{N}_{t+\Delta t}=\exp \left(-\frac{3 \mu \dot{\bar{\varepsilon}} \Delta t}{\bar{\sigma}}\right) \mathbf{N}_{t}+\left[1-\exp \left(-\frac{3 \mu \dot{\bar{\varepsilon}} \Delta t}{\bar{\sigma}}\right)\right] \sqrt{\frac{2}{3}} \frac{\mathbf{d}}{\dot{\bar{\varepsilon}}}
$$

This expression has the appropriate limits of being the flow potential normal at time $t$ for small strain increments, and it is in the direction of the applied strain for strain increments significantly larger than the limiting elastic strain. By comparison with Eq (16), the error is in the path that the normal takes between these two limits. The deviation peaks when the plastic strain increment is on the order of the elastic strain at yield.

It is important to note that Eq (17) is proposed to be the normal to the flow surface at the end of the time increment. It is not an average plastic flow direction over the time increment, and its intended use is different. The strategy adopted here is to find the stress direction corresponding to this flow potential normal. The magnitude of the stress is then adjusted to satisfy the yield function. This algorithm should result in fairly accurate stress predictions at large strain increments where the error in the normal calculation is small.

There are several significant details that need to be addressed. One is determination of the argument of the exponential in Eq (17) prior to knowing the plastic strain rate, and another is in determining the magnitude of the plastic strain rate needed for rate dependent material models and to advance the strain hardening. The solutions proposed here are not unique, but they are conceptually straight forward.

The argument of the exponential function can be estimated using the overstress relation from J2 plasticity

$$
-\frac{3 \mu \dot{\bar{\varepsilon}} \Delta t}{\bar{\sigma}}=1-\frac{\sigma_{e}^{T}}{\bar{\sigma}}
$$

where $\sigma_{e}^{T}$ is the effective trial stress computed from the yield function assuming there is no plasticity over the time increment. Since the equation for the normal is approximate, additional errors of a few percent in the argument of the exponential should not be of significant additional consequence. Given the quantity of information available at this state of the calculation, there are few viable alternatives.

Once the normal at the end of the time increment is determined, the direction of the stress tensor at the end of the time step can be determined by inverting the flow potential relation. The stress can then be placed in the form

$$
\boldsymbol{\sigma}_{t+\Delta t}=\bar{\sigma}_{t+\Delta t} \beta \mathbf{S}
$$


Here $\mathbf{S}$ is a second order unit tensor coaxial with the stress. It assumed that the yield function is homogeneous of degree one in stress so that the magnitude of the stress can be factored out as a scalar. Here $\bar{\sigma}$ is the material strength as a function of strain rate and temperature at the end of the time increment that appears in the yield function. $\beta$ is a factor that contains the orientation dependence of the yield function. If the yield function is written as $\mathcal{F}(\boldsymbol{\sigma})=\bar{\sigma}$ then

$$
\beta \mathcal{F}(\mathbf{S})=1
$$

For the generalized quadratic function this can be done analytically. For other flow potentials an iterative solution may be required.

Several options exist for determining an effective plastic strain rate, $\dot{\bar{\varepsilon}}$, consistent with the material hardening model and Eq (19). Perhaps the most physically appealing is through use of an energy balance where the change in energy is due to a sum of elastic and plastic parts. This insures that the effective plastic strain rate is work conjugate to the effective stress. Taking a central difference

$$
\frac{1}{2}\left(\boldsymbol{\sigma}_{t}+\boldsymbol{\sigma}_{t+\Delta t}\right): \Delta \varepsilon=\frac{1}{2}\left(\bar{\sigma}_{t}+\bar{\sigma}_{t+\Delta t}\right) \dot{\bar{\varepsilon}} \Delta t+\frac{1}{2}\left(\boldsymbol{\sigma}_{t+\Delta t}: \mathcal{L}^{-1}: \boldsymbol{\sigma}_{t+\Delta t}-\boldsymbol{\sigma}_{t}: \mathcal{L}^{-1}: \boldsymbol{\sigma}_{t}\right)
$$

Making the substitution from Eq (19) and rearranging, this becomes

$$
\bar{\sigma}^{2} \beta^{2} \mathbf{S}: \mathcal{L}^{-1}: \mathbf{S}+\bar{\sigma} \dot{\bar{\varepsilon}} \Delta t+\bar{\sigma}_{t} \dot{\bar{\varepsilon}} \Delta t-\bar{\sigma} \beta \mathbf{S}: \Delta \varepsilon-\boldsymbol{\sigma}_{t}: \Delta \boldsymbol{\varepsilon}-\boldsymbol{\sigma}_{t}: \mathcal{L}^{-1}: \boldsymbol{\sigma}_{t}=0
$$

This equation can be solved iteratively with the material strength model connecting the effective stress to the effective plastic strain rate. The difficulty with this approach is the lack of a direct connection to the yield function, and integration errors lead to inconsistencies in yield behavior. For near neutral loading, where the deformation is primarily tangential to the yield surface, small negative plastic strain rates are sometimes computed. The error can be traced to errors in the path dependence of the integral for the total energy increment as this quantity is less than the change in elastic stored energy.

To avoid difficulties at very small plastic strain increments, treatment of yielding and the plastic strain rate need to be consistent with the early assessment of whether or not the material is plastically deforming. This early decision involves the trial stress, so basing the plastic strain rate on the trial stress will avoid contradictory behavior. Following the radial return concepts for J2-flow theory, the flow strength and strain rate at the end of the time step can be determined from

$$
\bar{\sigma}(\dot{\bar{\varepsilon}}, T, \ldots)+3 \mu \dot{\bar{\varepsilon}} \Delta t-\sigma_{e}^{T}=0
$$

While there is no guarantee that the plastic strain rate computed in this manner is work conjugate to the flow stress, it is likely close. This will be investigated in future work.

With the flow strength computed, the updated stress follows from Eq (19) and all information needed for an explicit calculation has been determined. For implicit calculations additional computations are needed to compute the moduli. This is tedious, but straight forward. 


\section{Preliminary isotropic calculations}

Preliminary calculations were run to assess the stability and accuracy of the integration. The model implemented is the generalized quadratic yield function. The constants were chosen to provide an isotropic response, and results are compared to those of J2-Flow theory using the standard radial return algorithm. The calculations are run implicitly at a constant time step, and the boundary conditions imposed produce a load path change test.

A single element unit cube is subjected to uniaxial stress extension along the z-axis at a constant strain rate of 0.02 for 5 seconds. At that time those boundary conditions are removed and replace by simple shear deformation applied at all nodes shearing the nodes on the $\mathrm{y}$ face in the positive $\mathrm{x}$ direction. The total simulation is for 15 seconds with constant time increments of $0.01,1.0$ and 5.0. Figure 1 shows stress time histories from the proposed algorithm compared to results using radial return at the 0.01 time step. They are coincident.

Figure 2 compares stress histories from the two methods at the significantly larger time increments of 1.0 and 5.0 with the baseline curve at the 0.01 time step. The points from the proposed algorithm fall nearly on the baseline curve while the results from the radial return method have increasing deviation with increased time steps.

\section{Preliminary anisotropic analyses}

A simple uniaxial tension calculation was run as a preliminary assessment of behavior for anisotropic materials. The constants were chosen such that the contraction in one lateral direction should be twice that in the orthogonal direction. The numerical solution captures this trend nicely.

While this is encouraging, further observation from numerical results and analysis of Eq (17) reveals an unacceptable deficiency. While the proposed normal direction produces the correct stress direction in the limit as the strain increment approaches zero, the change in stress direction with increasing strain is not correct. At small non-zero strains the plastic flow direction is not correct. Such errors at small strain increments are unacceptable as the results must approach the rate form of the equation as the time step goes to zero. Equation (17) will not result in proper time step convergence.

\section{Discussion}

Improved accuracy is expected from the new integration scheme for J2-Flow theory since the integration over the time increment is exact for this model. The standard radial return is a backward difference algorithm and only first order accurate. Although the closed form integration algorithm will provide more accurate response than radial return, it is unlikely to replace the familiar and efficient technique.

The stress solution in Eq (12) is the exact solution for the generalized quadratic yield function for a rate independent material with no strain hardening. Although it would be possible to implement this form, exponentiation of the fourth order tensor could be computationally expensive. The most efficient algorithm might be to perform the calculations in a reference frame corotational with the material. In this way the eigenvalue decomposition only needs to be done once and the exponentiation can be handled in the principle coordinates.

It may be possible to construct acceptable forms for the normal direction of more 
complex potentials in the spirit of Eq (16). However, for a more general model the fourth order $\mathcal{K}$ tensor would likely be replaced by a tensor function of stress. Such a form could not be decomposed into fixed principle coordinates, and the numerical implementation would be very costly. For this reason, an alternative generalization of Eq (16) is not pursued at this time.

While the integration scheme based on $\mathrm{Eq}$ (17) has been determined to be unsuitable for anisotrpoic material models because of issues at small strain increments, it will still provide a good estimate for the stress at very large strain increments. It can, therefore, be used in backward difference integration schemes to estimate the stress to begin the iteration. Finding a good initial guess for the stress is often the most difficult task for these implicit solutions, so estimating the stress direction through Eq (17) and the plastic flow potential could prove quite useful.

Acknowledgement

This work was performed under the auspices of the U.S. Department of Energy by University of California, Lawrence Livermore National Laboratory under Contract W7405-Eng-48. UCRL-TR-219523 


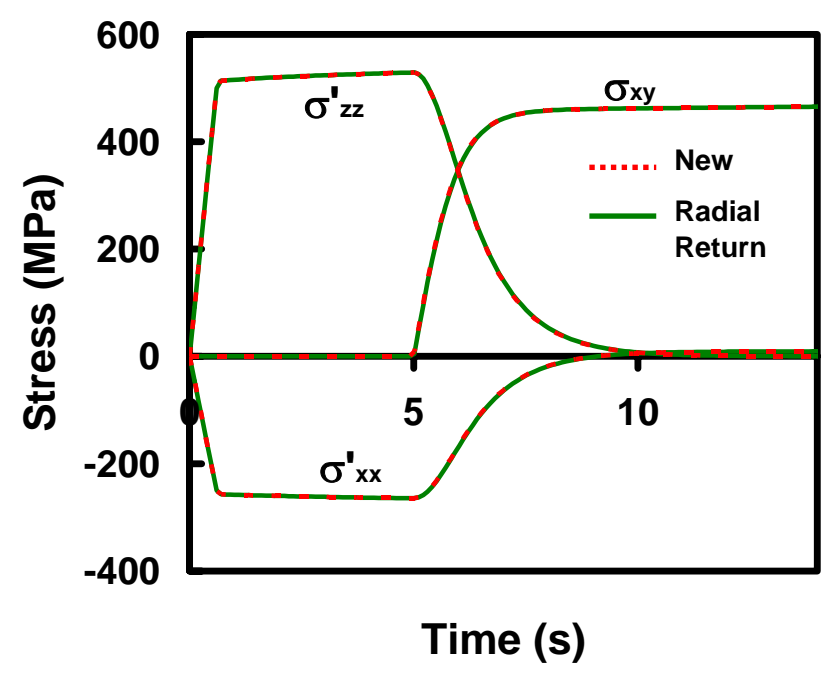

Figure 1. Comparison of stress response during load path change test for the new integration scheme and radial return with a time step of 0.01 .

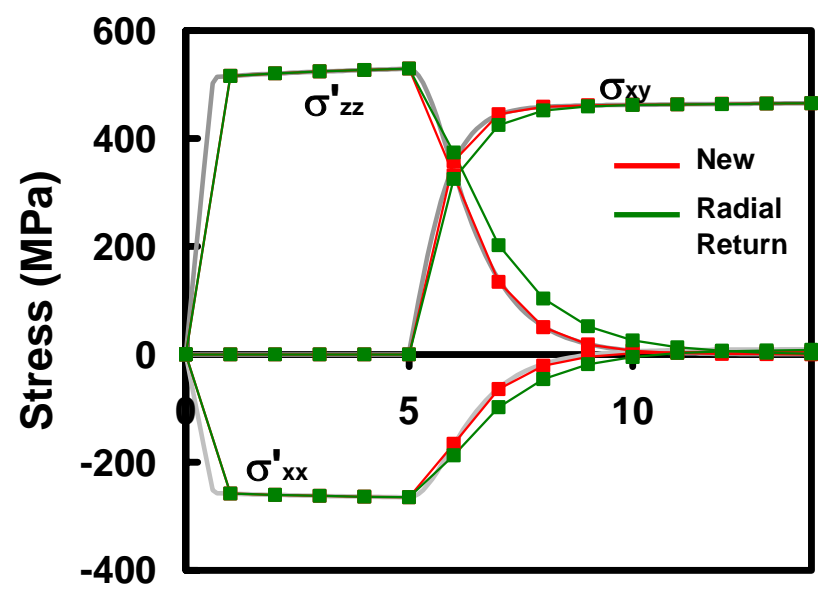

Time (s)

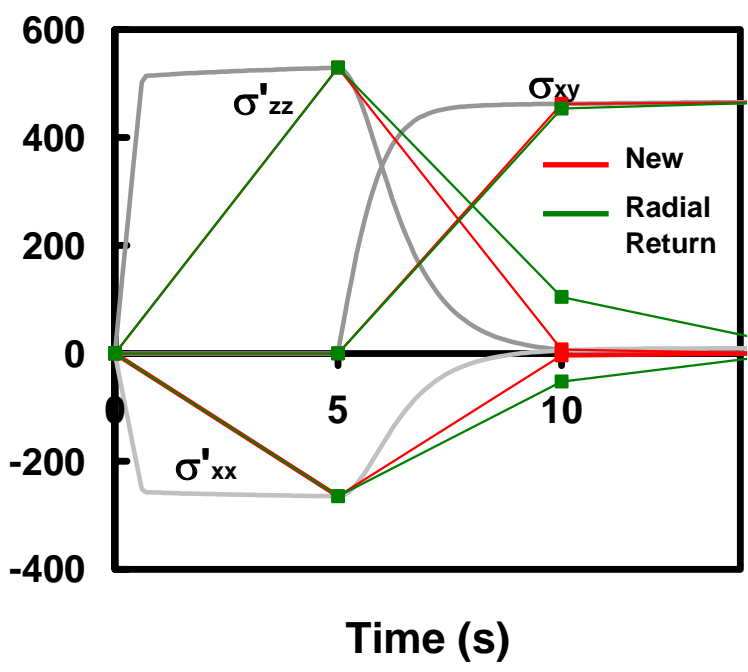

Figure 2. Comparison of stress response during load path change test for the new integration scheme and radial return for time steps of 1.0 and 5.0. The gray baseline curve is the same as in Figure 1. 